Akinkunmi and Lamikanra Afr. J. Infect. Dis. (2015) 9(2):67-72

http://dx.doi.org/10.4314/ajid.v9i2.10

\title{
A STUDY OF THE SUSCEPTIBILITY OF METHICILLIN RESISTANT COAGULASE-NEGATIVE STAPHYLOCOCCI ISOLATED FROM FAECAL SAMPLES OF CHILDREN TO COMMONLY USED ANTISEPTIC AGENTS
}

\author{
Ezekiel Olugbenga Akinkunmi*, Adebayo Lamikanra
}

\author{
Department of Pharmaceutics, Faculty of Pharmacy, Obafemi Awolowo University, Ile-Ife, Nigeria \\ *E-mail: akinkeroo@yahoo.com
}

\begin{abstract}
Background: This study was carried out to provide information on the susceptibility of methicillin resistant coagulase-negative staphylococci (MRCoNS) faecal isolates to some commonly used antiseptic agents.

Materials and Methods: For this purpose, 149 coagulase-negative staphylococci (CoNS) strains recovered from the faecal samples of children in IleIfe, a semi-urban settlement in Nigeria were screened against oxacillin by disc diffusion and agar screening methods. They were further screened against five antiseptic agents namely; benzalkonium chloride, cetrimide, chlorhexidine gluconate, gentian violet and acriflavine by the agar dilution method. Oxacillin resistant isolates were confirmed by screening for the mecA gene by the standard PCR method.

Results: Oxacillin resistant isolates were $30.2 \%$. While equal susceptibility was obtained for both MRCoNS and methicillin susceptible CoNS (MSCoNS) against gentian violet, reduced susceptibility to the order of one double dilution was obtained for MRCoNS against the remaining agents although the observed differences were not statistically significant $(\mathrm{p}>0.05)$. Isolates generally showed highest susceptibility to chlorhexidine gluconate $\left(\mathrm{MIC}_{50}=4 \mathrm{mg} / \mathrm{L} ; \mathrm{MIC}_{90}=32 \mathrm{mg} / \mathrm{L}\right)$ while they were found to be least susceptible to gentian violet $(\mathrm{MIC} 50=32 \mathrm{mg} / \mathrm{L} ; \mathrm{MIC} 90>32 \mathrm{mg} / \mathrm{L})$. $S$. hominis strains were the most susceptible to chlorhexidine gluconate $\left(\mathrm{MIC}_{50}<1 \mathrm{mg} / \mathrm{L} ; \mathrm{MIC}_{\text {mean }}=2.9 \mathrm{mg} / \mathrm{L}\right)$ whereas $S$. saprophyticus were the least susceptible to this agent $\left(\mathrm{MIC}_{50}=16 \mathrm{mg} / \mathrm{L} ; \mathrm{MIC}_{\text {mean }}=5.8 \mathrm{mg} / \mathrm{L}\right)$.

Conclusion: It is concluded that children feces can be an important source of MRCoNS with reduced susceptibility to some commonly used antiseptic agents, a source that appears to receive little attention and thus warrants further investigation.
\end{abstract}

Key words: surveillance, antimicrobials, drug resistance, efficacy, faeces, antiseptics

\section{Introduction}

Coagulase-negative staphylococci (CoNS) species which were found as contaminants of blood cultures and historically thought to be nonpathogenic, are increasingly associated with a variety of infections (Huebner and Goldmann, 1999). These infections have been reported to be refractory to therapy because of the prevalence of antibiotic resistance associated with CoNS strains involved in such infections (Archer and Climo, 1994).

Reports have shown that approximately $70 \%$ of $S$. epidermidis strains isolated in the hospital environment have acquired resistance to methicillin, and majority of them are also resistant to other antimicrobial agents (John et al., 2002; Miragaia et al., 2008). Other CoNS were also reported to have acquired resistance to many classes of antimicrobial agents (John et al., 2002; Chaudhury and Kumar, 2007). In the same vein, methicillin resistant Staphylococcus aureus (MRSA) has become a serious problem both in the hospitals and the communities worldwide and it is now a challenge for public health practitioners (Naimi et al., 2003).

Antiseptics play an essential role in reducing the number of transient microflora on the hand and the person to person transmission of antibiotic resistant and pathogenic microbes and are therefore used widely in the maintenance of hygiene (Boyce and Pittet, 2002). This being the case, there is growing interest in the potential of these agents to contribute to the emergence and dissemination of pathogenic and drug resistant microbes. There are also increasing reports of reduced susceptibility to these agents by these microbes. For example, increased Minimum Inhibitory Concentrations (MICs) for methicillin resistant Staphylococcus aureus (MRSA) strains have been reported for some antiseptics, including chlorhexidine, cetrimide, benzalkonium chloride and parahydroxybenzoates (Irizzary et al., 1998; Poole, 2002; Akinkunmi and Lamikanra, 2012). However, there is little information concerning the susceptibility to antiseptics by methicillin resistant CoNS which are of increasing clinical significance.

The main mechanism of methicillin resistance in staphylococci involves the expression of PBP2a encoded by the mec A gene. Nevertheless, other mechanism of resistance such as a decreased binding capacity of PBP3 (Petinaki et al., 2001) or the production of $\beta$-lactamase against methicillin and other penicillinase-resistant penicillins (Massidda et al., 1992) have been described. The reported link between the susceptibility of methicillin resistant staphylococci to antibiotics and other antimicrobial agents such as disinfectants and antiseptics was shown genetically to be a result of interplay between disinfectant resistance genes $(q a c A / B)$ and $\beta$-lactamase gene (bla Z) in staphylococci (Sidhu et al., 2000).

Although, the skin is widely known to be the main reservoir of CoNS, the gastrointestinal tract has been identified as another important reservoir of these organisms (Stiefel and Donskey, 2004; Akinkunmi and Lamikanra, 2010). Intestinal and perineal carriage of staphylococci have been implicated as important contributors to their environmental dissemination as patients often contaminate the adjacent environment. This is especially true in cases of diarrhoea where a high level of contamination of environmental surfaces can occur as a result of loose or liquid stools (Boyce et al., 2007).

In the present study, the susceptibility of methicillin resistant and methicillin susceptible CoNS isolates from faecal samples of children to some widely used antiseptic agents was examined in an attempt to bridge the knowledge gap presently occurring in this area. The study also attempt to provide information on the ability of these antiseptics to reduce the proliferation of these organisms and thus prevent their transmission from one person to another. 
http://dx.doi.org/10.4314/ajid.v9i2.10

\section{Materials and Methods \\ Epidemiological Data of Subjects}

CoNS isolates were from fecal samples obtained from 293 children of both sexes under 3 years of age, from Ile-Ife, a semi-urban settlement in Osun State of Nigeria and the adjoining villages. The children were among those attending immunisation clinics and day care centres in the community and from households selected randomly within Ile-Ife. All specimens were collected with the informed consent of the parents or guardians of the children and the study was approved by the Institutional Review Board of the participating institution. Fecal samples were collected, stored, and transported to laboratory as described in Akinkunmi and Lamikanra (2010).

\section{Bacteria strains}

CoNS isolates were identified by morphological and biochemical characteristics using standard methods described by Ieven et al. (1999) and used by Akinkunmi and Lamikanra (2010). The isolates were preserved in cryovials (Nalgene, Rochester, NY and USA) and stored at $-20^{\circ} \mathrm{C}$ and in agar stabs at $4^{\circ} \mathrm{C}$. Molecular confirmation of the phenotypic identification of CoNS was performed by PCR assays to confirm the absence of the nuc gene in these isolates as described previously (Akinkunmi and Lamikanra, 2010). All the CoNS strains (n=149) isolated from these fecal samples, including forty-five (45) S. epidermidis, twenty-six (26) S. haemolyticus, twenty-four (24) S. capitis, eleven (11) S. xylosus, eight (8) S. saprophyticus, eight (8) S. warneri, six (6) S. hominis, five (5) S. schleiferi, three (3) S. capitis sub ureolyticus, three (3) S. lugdunensis and ten (10) unspeciated CoNS were used in this study.

\section{Media, Antibiotics and Antiseptics}

All chemicals and reagents used were of pure analytical grade. Oxacillin discs and powder were obtained from Oxoid, England, while Mueller Hinton Agar was from Remel, England. Acriflavine and Cetrimide were obtained from Hopkin and Williams, England, Benzalkonium chloride and Gentian violet from BDH, England, while Chlorhexidine gluconate was obtained from Fluka Chemika, Switzerland.

\section{Determination of oxacillin susceptibility \\ Disk diffusion method}

Eighteen to 24-hour old colonies from a plate inoculated from a single colony were used to prepare the inoculum for all systems. Oxacillin disk diffusion susceptibility testing was performed with $1-\mu \mathrm{g}$ oxacillin disc. The test was carried out on Mueller-Hinton agar using inocula equivalent to $0.5 \mathrm{McFarland}$ barium sulphate standard unit (average turbidity, $10^{8} \mathrm{cfu} / \mathrm{ml}$ ) following the recommendations of the Clinical and Laboratory Standards Institute (CLSI) (2008). The diameters of inhibition zones were measured in millimeters and interpreted using the Progressive Diagnostics Manufacturers (PDM) Interpretive Charts (AB Biodisc, Solna, Sweden); this procedure agrees with the Clinical Laboratory Standard Institute (CLSI) [16] guidelines. S. aureus NCTC 6571 was used as control.

\section{Agar screening method}

Oxacillin-salt agar (Mueller-Hinton agar containing $4 \% \mathrm{NaCl}$ and $6 \mathrm{mg}$ oxacillin $\mathrm{L}^{-1}$ ), was used for the agar screening method as recommended by the CLSI (2008). Inoculation of the oxacillin-salt agar plates was performed by using a multipoint inoculator. Any isolate which grew on the test medium within 48 hours at $35^{\circ} \mathrm{C}$ was regarded as being oxacillin resistant. The experiment was performed in duplicate. S. aureus (MRSA) ATCC R 43300 was used as the control.

\section{Molecular detection of the mecA gene by PCR}

The presence of the mecA gene responsible for the production of PBP2A was screened in all CoNS isolates found to be resistant to oxacillin by the disc diffusion and agar-screening techniques as previously described by Akinkunmi and Lamikanra (2012b).

\section{Determination of susceptibility of CoNS to antiseptics}

Stock concentrations of each antiseptic agent were freshly prepared. Minimum inhibitory concentrations to the antiseptics; benzalkonium chloride $\left(0.5-32 \mu \mathrm{gml}^{-1}\right)$, chlorhexidine gluconate $\left(0.5-32 \mu \mathrm{gml}^{-1}\right)$, cetrimide $\left(0.5-32 \mu \mathrm{gml}^{-1}\right)$, gentian violet $\left(0.5-32 \mu \mathrm{gml}{ }^{-1}\right)$ and acriflavine $(0.5$ $512 \mu \mathrm{gml}^{-1}$ ) were determined by the agar doubling dilution method according to CLSI guidelines (2008). Plates containing $10 \mathrm{~mL}$ of test medium incorporating doubling dilutions of antimicrobial agents were prepared and dried. Plates containing no antimicrobials were included as controls. Inocula of organism equivalent to $0.5 \mathrm{McF}$ arland were prepared and applied to the surface of plates containing antiseptics and control plates with a multipoint innoculator. The plates were allowed to dry at room temperature for 15 minutes before incubating for 48 hours at $35^{\circ} \mathrm{C}$. The lowest concentration of antiseptic inhibiting growth was taken to be the MIC.

\section{Statistical Analysis}

Chi-square test or the Fisher exact test was used in determining probabilities and level of significance. All hypotheses were considered significant if $\mathrm{p}<0.05$. The analysis was performed using SPSS Version 12 statistical software (IBM, Chicago, USA). 
http://dx.doi.org/10.4314/ajid.v9i2.10

\section{Results}

Subjects

Of the 293 children from whom faeces were collected, 165 (56.3\%) were male. CoNS were isolated from 118 (40.3\%) of the children. A significantly greater number of S. epidermidis was isolated from male $(73.3 \%)$ than female $(26.7 \%)$ children (p < 0.05$)$. For the other CoNS species, there was no significant relationship between faecal carriage and children characteristics such as the age and gender of the children $(p>0.05)$.

\section{Oxacillin susceptibility of the CoNS isolates}

Forty-five (30.2\%) of the 149 CoNS isolates were found to be oxacillin resistant. The disc-diffusion test using oxacillin discs as well as the oxacillin agar plate screening method gave the same results and these were confirmed by the detection of the mecA gene by the polymerase chain reaction. All strains with MIC of oxacillin $\geq 6 \mu \mathrm{g} / \mathrm{mL}$ as indicated in the oxacillin agar plate screening results were regarded as MRCoNS.

\section{Susceptibility of CoNS strains to antiseptics}

The MICs at which 50 and $90 \%$ of CoNS isolates were inhibited ( $\mathrm{MIC}_{50}$ and $\mathrm{MIC}_{90}$, respectively) by antiseptics for MRCoNS and MSCoNS were noted. MRCoNS showed a comparatively higher MIC to antiseptics compared with MSCoNS (Table 1).

Table 1: Antiseptic susceptibility of MRCoNS and MSCoNS isolates

\begin{tabular}{llll}
\hline Staphylococci (No of isolates) & Antimicrobial agents & MIC $(\mathbf{m g} / \mathbf{L})$ \\
\hline \multirow{3}{*}{ MRCoNS (45) } & & $\mathbf{M I C}_{\mathbf{5 0}}$ & $\mathbf{M I C}_{\mathbf{9 0}}$ \\
\cline { 2 - 3 } & Benzalkonium chloride & 8 & $>32$ \\
& Chlorhexidine gluconate & 8 & 32 \\
& Cetrimide & 32 & $>32$ \\
Gentian Violet & 32 & $>32$ \\
MSCoNS (104) & Acriflavin & 16 & 256 \\
& Benzalkonium chloride & 4 & 32 \\
& Chlorhexidine gluconate & 4 & 32 \\
& Cetrimide & 16 & $>32$ \\
Total CoNS (149) & Gentian Violet & 32 & $>32$ \\
& Acriflavin & 8 & 64 \\
& Benzalkonium chloride & 8 & $>32$ \\
& Chlorhexidine gluconate & 4 & 32 \\
& Cetrimide & 32 & $>32$ \\
& Gentian Violet & 32 & $>32$ \\
& Acriflavin & 16 & 128 \\
\hline
\end{tabular}

MRCoNS=Methicillin Resistant Coagulase Negative Staphylococci;

MSCoNS= Methicillin Susceptible Coagulase Negative Staphylococci;

Results also showed that the CoNS isolates generally showed highest susceptibility to chlorhexidine gluconate and were least susceptible to gentian violet (Table 2). S. hominis strains were found to be the most susceptible to chlorhexidine $\left(\mathrm{MIC}_{50}<1 \mathrm{mg} / \mathrm{L} ; \mathrm{MIC}\right.$ mean $\left.=2.9 \mathrm{mg} / \mathrm{L}\right)$ whereas $S$. saprophyticus were the least susceptible to this agent $\left(\mathrm{MIC}_{50}=16 \mathrm{mg} / \mathrm{L} ; \mathrm{MIC}_{\text {mean }}=5.8 \mathrm{mg} / \mathrm{L}\right)$ (Figure 1$)$.

Table 2: Antiseptic susceptibility of individual CoNS isolates

\begin{tabular}{|c|c|c|c|c|c|c|c|c|c|c|}
\hline \multirow{3}{*}{ Organisms (No) } & \multicolumn{10}{|c|}{ Antimicrobial agents (MIC, mg/L) } \\
\hline & \multicolumn{2}{|c|}{$\begin{array}{l}\text { Benzalkonium } \\
\text { chloride }\end{array}$} & \multicolumn{2}{|c|}{ Chlorhexidine } & \multicolumn{2}{|c|}{ Cetrimide } & \multicolumn{2}{|c|}{ Gentian violet } & \multicolumn{2}{|c|}{ Acriflavine } \\
\hline & $\mathrm{MIC}_{50}$ & $\mathrm{MIC}_{90}$ & $\mathrm{MIC}_{50}$ & $\mathrm{MIC}_{90}$ & $\mathrm{MIC}_{50}$ & $\mathrm{MIC}_{90}$ & $\mathrm{MIC}_{50}$ & $\mathrm{MIC}_{90}$ & $\mathrm{MIC}_{50}$ & $\mathrm{MIC}_{90}$ \\
\hline S. haemolyticus (26) & 16 & $>32$ & 16 & 32 & 32 & $>32$ & 16 & $>32$ & 32 & 512 \\
\hline S. capitis (24) & 8 & $>32$ & 2 & 32 & 32 & $>32$ & 32 & $>32$ & 16 & 128 \\
\hline S. xylosus (11) & 4 & $>32$ & 4 & 32 & 8 & $>32$ & $>32$ & $>32$ & 16 & 128 \\
\hline Other CoNS (43) & 16 & 32 & 4 & 16 & 16 & $>32$ & 32 & $>32$ & 16 & 128 \\
\hline
\end{tabular}




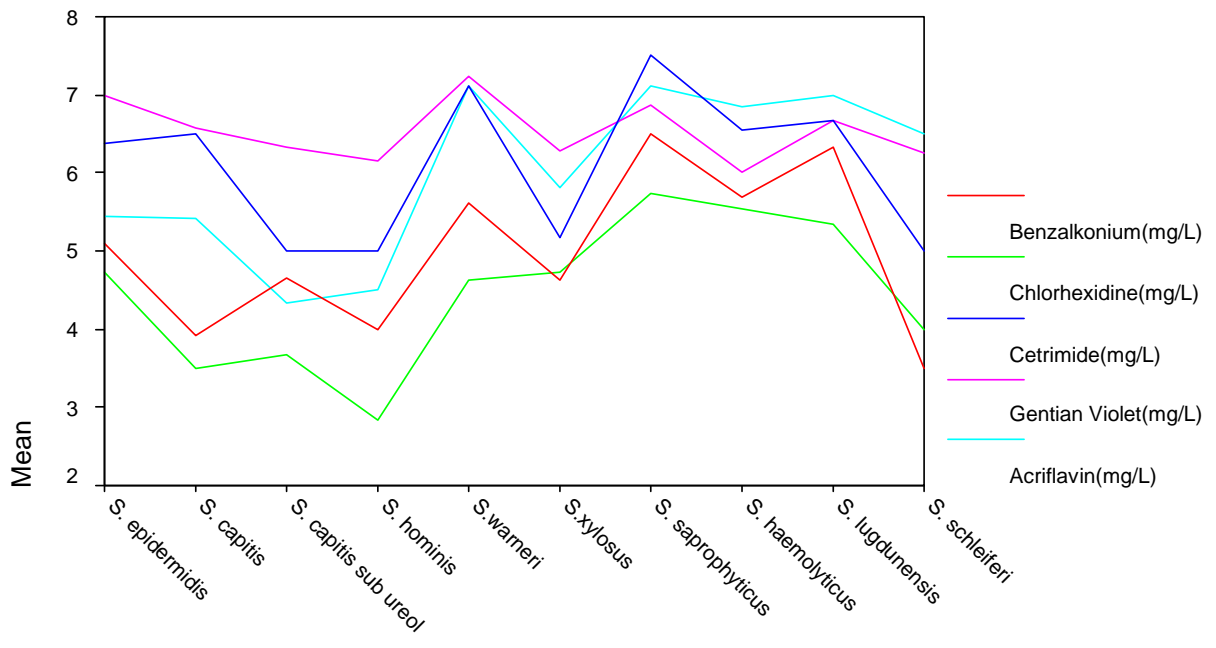

isolate name

Figure 1: Susceptibility of different staphylococci species to antiseptics: Mean MIC (mg/L) to which organisms are susceptible.

\section{Discussion}

Although microbial resistance to germicides such as antiseptics, disinfectants, and preservatives has been widely reported in literature (Russell, 2001; Poole, 2002; Akinkunmi and Lamikanra, 2012) no studies screening germicides against CoNS isolates from fecal samples were found in literature. Earlier studies have indicated that feces are prominent sources of multidrug resistant CoNS (Dominquez et al., 2002; Stiefel and Donskey, 2004; Akinkunmi and Lamikanra, 2010). This study therefore provides a baseline data for the susceptibility of strains of these organisms to antiseptic agents.

The susceptibility of CoNS isolates from the faeces of children in Ile-Ife and its environs to cationic antiseptic agents; benzalkonium chloride, cetrimide and chlorhexidine gluconate commonly used for the disinfection of skin and hands in the study area was determined. Although dyes such as gentian violet (a triphenylmethane dye) and acriflavine (an acridine dye) are no more recommended for use in antisepsis because of their reported association with toxicity to mucus membranes and their carcinogenic potentials (Drinkwater, 1990), they are still being used in the study area for the treatment of minor cuts and abrasions on children and were thus included in the screening experiments.

All the CoNS species investigated in this study have been previously reported to be clinically important (Kloos and Bannerman, 1999) and were also reported to be increasingly associated with both community and nosocomial infections as well as with resistance to antimicrobial agents (Huebner and Goldmann, 1999; Akinkunmi and Lamikanra, 2010). Over 30\% of the 149 faecal isolates of CoNS used in this study were found to be oxacillin resistant, a result that corroborates the literature reports. The high prevalence of methicillin resistance in the CoNS isolates from the sampled children also corroborates reports of multiply antibiotic resistant pathogens already circulating among the populations in the study area (Okeke et al., 1999; Okeke and Sosa, 2003). Furthermore, in the case of S. aureus, intestinal colonization by staphylococci has been reported to be associated with increased frequency of staphylococci in the skin of hospital patients resulting in increased skin infections (Bhalla et al., 2007).

With the MICs of gentian violet against both MRCONS and MSCoNS strains being of equal values $\left(\mathrm{MIC}_{50}=32 \mathrm{mg} / \mathrm{L}\right.$ and $\left.\mathrm{MIC} 90>32 \mathrm{mg} / \mathrm{L}\right)$, the results of the study indicate that the susceptibilities of these strains to this antiseptic agent were the same. In other cases, as found for benzalkonium chloride, chlorhexidine gluconate, cetrimide and acriflavine, the $\mathrm{MIC}_{50} \mathrm{~s}$ of $\mathrm{MRCoNS}$ were found to be one double dilution lower than that for MSCoNS indicating comparatively lower susceptibility to these agents. However, the differences in the susceptibilities to these agents were not found to be statistically significant $(\mathrm{p}>0.5$ ). In the case of chlorhexidine, this corroborates the results of Mengistu et al. (1999) who could not detect any relationship between methicillin resistance and susceptibility to chlorhexidine for their 17 CoNS clinical isolates.

This study showed that the MICs of gentian violet, cetrimide and acriflavine against these organisms are much higher than those of chlorhexidine gluconate and benzalkonium chloride. This indicated that the use of the former agents for skin disinfections, such as the treatment of wounds, should be discouraged not only on the basis of their reported toxicity but also on the basis of the possibility of their ineffectiveness. Instead, more effective antiseptics like benzalkonium chloride but much more importantly chlorhexidine gluconate should be used for the disinfection of skin and mucous membranes as well as general disinfection to ensure the elimination of resistant strains of these organisms and the prevention of crossinfection. The effectiveness of chlorhexidine gluconate as an antiseptic is well documented in the literature (Mullany et al., 2006; Soofi et al., 2012). Combinations of chlorhexidine with other effective antiseptic agents such as alcohols, phenolics, halogens or the quarternary ammonium salts have also been found beneficial (Boyce and Pittet, 2002).

It is important to educate patients and health care workers on strategies to limit the spread of infections due to MRCoNS. There should be proper environmental cleaning and decontamination of equipments with appropriate antimicrobial agents. Hospital policies should also include the importance of training in infection control such as proper hand-washing with effective antiseptics. 
http://dx.doi.org/10.4314/ajid.v9i2.10

\section{Conclusion}

It is concluded that the preponderance of MRCoNS in the faeces of children is of crucial importance to health care. Children faeces can be an important source of MRCoNS with reduced susceptibility to some commonly used antiseptic agents, a source that appears to receive little attention and thus warrants further investigation.

\section{Acknowledgements}

We thank the Management and staff of the institutions where samples were collected for support and technical assistance. The abstract of this work has been partly published in the abstract book of the Third international Conference for Improving Use of Medicines: Informed Strategies, Effective Policies, Lasting Solutions held in November 14-18, 2011 in Antalya, Turkey, Abstract No 848 page 224.

Conflict of interest: None to declare.

\section{References}

1. Akinkunmi, E.O. and Lamikanra, A (2010). Species distribution and antibiotic resistance in coagulase negative staphylococci colonizing the gastrointestinal tract of children in Ile-Ife, Nigeria. Trop J Pharm Res 9(1):35-43.

2. Akinkunmi, E.O. and Lamikanra, A. (2012). Susceptibility of community associated methicillin resistant Staphylococcus aureus isolated from faeces to antiseptics. J Inf Dev Count 6(4): 317-323.

3. Akinkunmi, E.O. and Lamikanra, A (2012b). A study of the gastrointestinal carriage of antibiotic resistant Staphylococcus aureus by Nigerian children. African Health Science, 12(3):381-388.

4. Archer, G. and Climo, M.W. (1994). Antimicrobial susceptibility of coagulase-negative staphylococci. Antimicrob Agents Chemother 38:22312237.

5. Bhalla, A., Aron, D.C. and Donskey, C.J. (2007). Staphylococcus aureus intestinal colonization is associated with increased frequency of S. aureus on skin of hospitalized patients. BMC Infect Dis 7:105-109.

6. Boyce, J.M. and Pittet, D. (2002). Guideline for hand hygiene in health-care settings. Recommendations of the Healthcare Infection Control Practices Advisory Committee and the HICPAC/SHEA/APIC/IDSA Hand Hygiene Task Force. Society for Healthcare Epidemiology of America/Association for Professionals in Infection Control/Infectious Diseases Society of America. Morbid Mortal Wkly Rep Recomm Rep 51:1-4.

7. Boyce, J.M., Havill, N.L., Otter, J.A. and Adams, N.M. (2007). Widespread environmental contamination associated with patients with diarrhea and methicillin-resistant Staphylococcus aureus colonization of the gastrointestinal tract. Infect Control Hosp Epidemiol 28:1142-1147.

8. Chaudhury, A. and Kumar, A.G. (2007). In vitro activity of antimicrobial agents against oxacillin resistant staphylococci with special reference to Staphylococcus haemolyticus. Indian J Medical Microbiol. 25(1):50-52.

9. Clinical and Laboratory Standards Institute. Performance standards for antimicrobial susceptibility testing; 16th informational supplement. M100-S16. Clinical and Laboratory Standards Institute, Wayne, Pa. 2008.

10. Domínguez, E., Zarazaga, M. and Torres C. (2002). Antibiotic Resistance in Staphylococcus Isolates Obtained from Fecal Samples of Healthy Children. J Clin Microbiol 40:2638-2641.

11. Drinkwater, P. (1990). Gentian violet - is it safe? [editorial]. Aust N Z Obstet Gynecol 30:65-66.

12. Huebner, J. and Goldmann, D.A. (1999). Coagulase-negative staphylococci: role as pathogens. Annu Rev Med 50:223-236.

13. Ieven, M., Verhoeven, J., Pattyn, S.R. and Goossens, H. (1999). Rapid and economical method for species identification of clinically significant coagulase-negative staphylococci. J Clin Microbiol 33:1060-1063.

14. Irizzary, L., Merlin, T., Rupp, J. and Griffith, J. (1998). Reduced susceptibility of methicillin resistant Staphylococcus aureus to cetylpyridinium chloride and chlorhexidine. Chemotherapy 42 (4):248-252.

15. John, M.A., Pletch, C. and Hussain, Z. (2002). In vitro activity of quinupristin/ dalfopristin, linezolid, telithromycin and comparator antimicrobial agents against 13 species of coagulase-negative staphylococci. J Antimicrob Chemother 50:933-938.

16. Kloos, W.E. and Bannerman, T.L. (1999). Update on clinical significance of coagulase-negative staphylococci. Clin Microbiol Rev 7:117-40.

17. Massidda, O., Montanari, M.P. and Varaldo, P.E. (1992). Evidence for a methicillin-hydrolysing beta-lactamase in Staphylococcus aureus strains with borderline susceptibility to this drug. FEMS Microbiol Lett 71:223-227.

18. Mengistu, Y., Ergie, W. and Bellete, B. (1999). In vitro suceptibility of staphylococci to chlorhexidine and antibiotics. Ethiop J Health Dev 13(3):223-228.

19. Miragaia, M., Carric s, J.A., Thomas, J.C., Couto, I., Enright, M.C. and de Lencastre, H. (2008). Comparison of Molecular Typing Methods for Characterization of Staphylococcus epidermidis: Proposal for Clone Definition. Journal of Clinical Microbiology 46 (1):118-129.

20. Mullany, L.C., Darmstadt, G.L. and Tielsch, J.M. (2006). Safety and impact of chlorhexidine antisepsis interventions for improving neonatal health in developing countries. Pediatr Infect Dis J 25: 665-75.

21. Naimi, T.S., LeDell, K.H., Como-Sabetti, K.M. et al. (2003). Comparison of community and healthcare associated methicillin-resistant Staphylococcus aureus infection. JAMA 290: 2976-84.

22. Petinaki, E., Dimitracopoulos, G. and Spiliopoulou, I. (2001). Decreased affinity of PBP3 to methicillin in a clinical isolate of Staphylococcus epidermidis with borderline resistance to methicillin and free of the mecA gene. Microb Drug Resist 7:297-304.

23. Okeke, I.N. and Sosa, A. (2003). Antibiotic resistance in Africa - discerning the enemy and plotting a defence. Africa Health:10-15.

24. Okeke, I.N., Lamikanra, A. and Edelman, R. (1999). Socioeconomic and behavorial factors leading to acquired bacterial resistance to antibiotics in developing countries. Emerg Infect Dis 5:18-27.

25. Poole, K. (2002). Mechanisms of bacterial biocide and antibiotic resistance. J Appl Microbiol 92:55S-64S.

26. Russell, A.D. (2001). Mechanisms of bacterial insusceptibility to biocides. Am J Infect Control 29:259-261. 
http://dx.doi.org/10.4314/ajid.v9i2.10

27. Sidhu, M.S., Heir, E., Sorum, H. and Holck, A. (2000). Linkage between Penicillin and Benzalkonium Chloride Resistance in Staphylococci from Clinical and Food Environments. Abstr Intersci Conf Antimicrob Agents Chemother 40:131.

28. Soofi, S., Cousens, S., Imdad, A., Bhutto, N., Ali, N. and Bhutta, Z.A. (2012). Topical application of chlorhexidine to neonatal umbilical cords for prevention of omphalitis and neonatal mortality in a rural district of Pakistan: a community-based, cluster-randomised trial. Lancet; published online Feb 8. DOI:10.1016/ S0140-6736(11)61877-1.

29. Stiefel, U. and Donskey, C. J. (2004). The role of the intestinal tract as a source for transmission of nosocomial pathogens. Current Infectious Disease Reports 6: 420-425. 